\title{
A Sinc Approximation for the Indefinite Integral
}

\author{
By Ralph Baker Kearfott
}

\begin{abstract}
A method for computing $\int_{0}^{x} f(t) d t, x=(0,1)$ is outlined, where $f(t)$ may have singularities at $t=0$ and $t=1$. The method depends on the approximation properties of Whittaker cardinal, or sinc function expansions; the general technique may be used for semi-infinite or infinite intervals in addition to $(0,1)$. Tables of numerical results are given.
\end{abstract}

1. Introduction and Summary. Here we present a basic method and experimental results for approximating $F(x)=\int_{0}^{x} f(t) d t, x \in(0,1)$, where $f$ is smooth, but may have singularities at $t=0$ or $t=1$. The method is based on approximation properties of sinc functions

$$
S(k, h)(x)=\frac{\sin [(\pi / h)(x-k h)]}{(\pi / h)(x-k h)} .
$$

With minor modifications, the method may also be employed to compute $\int_{a}^{x} f(t) d t$, $t \in(a, b)$, where $a, b$ or both $a$ and $b$ are possibly infinite.

Our underlying formula is similar to formula (3.36) in [4], given there without proof. Our formula is considered in detail here, is proven, and is backed by numerical experiments. (See Section 4 for more discussion.)

In Section 2 we give the relevant properties of sinc functions, assumptions, and basic techniques. (Such techniques appear in more detail in [4] and elsewhere.) In Section 3 we present and verify our integral approximation formulas. Four tables of experimental results and conclusions appear in Section 4.

2. Underlying Properties and Assumptions. A general review of sinc functions and their uses has recently been given by Stenger in [4]. We therefore only outline properties important to our present goals, and refer the reader to [4] for further references.

Approximations on $(0,1)$ are obtained from corresponding approximations on $\mathbf{R}=(-\infty, \infty)$ via a conformal map. To be approximable on $\mathbf{R}, f \in C^{\infty}(\mathbf{R})$ must obey certain analyticity and boundedness conditions in a strip in the complex plane C which contains $\mathbf{R}$. Through the conformal map, we obtain a corresponding "eye-shaped" region (cf. Figure 4.1, p. 185, of [4]) containing the interval $(0,1)$, in which our integrands must obey certain analyticity and boundedness conditions. With this in mind, we first list properties over $\mathbf{R}$.

Received April 23, 1981: revised March 10, 1982.

1980 Mathematics Subject Classification. Primary 65D30; Secondary 41-04, 41A25, 41A55.

Key words and phrases. Indefinite integrals, singular integrals, quadrature, sinc functions, Whittaker's cardinal function, approximation theory. 
Defintion 2.1. Let $d>0$ and let 12 , denote the open strip

$$
\mathrm{oI}_{d}=\{z \in \mathbf{C}:|\operatorname{Im}(z)|<d\} \text {. }
$$

Let $B_{p}\left((0)_{d}\right)$ denote the family of all functions $f$ that are analytic in $(n)$ and which satisfy

$$
\int_{-d}^{d}|f(x+i y)| d y \rightarrow 0 \quad \text { as } x \rightarrow \pm \infty
$$

and such that

$$
N_{p}\left(f, \mathrm{Q}_{d}\right)=\lim _{y \rightarrow d^{-}}\left\{\left(\int_{\mathbf{R}} \mid f(x+i y) P d x\right)^{1 / p}+\left(\int_{\mathbf{R}}|f(x-i y)|^{p} d x\right)^{1 / p}\right\}<\infty .
$$

We denote $B_{1}\left(\mathcal{N}_{d}\right)$ by $B\left(\mathcal{N}_{d}\right)$; in all results below, $p=1$ for simplicity unless otherwise stated, although most of these results generalize to arbitrary $p$; cf. [4, Section 3.1].

If $f \in B\left(\Phi_{d}\right)$, we approximate $f$ by a truncated "Whittaker cardinal series"

$$
f(x)=C_{N}(f, h)(x)=\sum_{k=-1}^{N} f(k h) S(k, h)(x)
$$

where $S(k, h)(x)$ is as in (1.1). We state the following without proof:

Theorem 2.1. (See [2, pp. 237-238], and [4, pp. 177-178].) Suppose $f \in B_{p}\left({ }^{(0)} d\right)$ for some $p \in[1, \infty)$, and suppose

$$
|f(x)| \leqslant C e^{-\alpha|x|} \text { for all } x \in \mathbf{R}\left(\text { i.e., }|f(x)|=O\left(e^{-\alpha|x|}\right)\right)
$$

for some $C$ and $\alpha>0$ dependent on $f$. Choose $h=[\pi d /(\alpha N)]^{1 / 2}$. Then there is a $C^{\prime}$ dependent on $f$, but not on $N$, such that

$$
\left\|f(x)-C_{N}(f, h)(x)\right\|_{x} \leqslant C^{\prime} N^{1 / 2} e^{-(\pi d \alpha N)^{1 / 2}},
$$

where $\|\cdot\|_{x}$ is the supremum norm over $\mathbf{R}$.

Here we need not only to approximate functions $f$, but also their integrals. We have

TheOREM 2.2. (Trapezoidal Rule; cf. e.g. [2, p. 229], or [4, p. 178].) Suppose $f \in B\left(\mathscr{D}_{d}\right)$, suppose $f$ satisfies $(2.5)$, and choose $h^{\prime}=[2 \pi d / \alpha N]^{1 / 2}$. Then

$$
\left|\int_{-\infty}^{\infty} f(t) d t-h^{\prime} \sum_{k=-N}^{N} f\left(k h^{\prime}\right)\right| \leqslant C_{I} e^{-(2 \pi d \alpha N)^{1 / 2}}
$$

for some integral approximation constant $C_{I}$ dependent on $f$ but not on $N$.

To obtain approximations over $(0,1)$, we make use of the following conformal map:

$$
\varphi(z)=\log \left(\frac{z}{1-z}\right), \quad \varphi^{\prime}(z)=\frac{1}{z(1-z)} .
$$

For any $d$ such that $0<d \leqslant \pi / 2, \varphi$ maps the region

$$
\tilde{\mathscr{O}}_{d}=\{z:|\arg [z /(i-z)]|<d\}
$$


onto $\mathscr{D}_{d}$ where $\mathscr{D}_{d}$ is as in (2.1). (Note that $\partial \tilde{\mathscr{D}}_{d}$ consists of two circular arcs intersecting symmetrically with an angle of $2 d$ at 0 and 1); (cf. [4, p. 185].) Define

$$
z=\psi(w)=\varphi^{-1}(w)=\frac{e^{w}}{1+e^{w}}, \quad \psi^{\prime}(w)=\psi(w)[1-\psi(w)] .
$$

Suppose $f$ is defined and analytic in the interior of $\tilde{\mathscr{D}}_{d}$, and define

$$
\tilde{f}(w)=f(\psi(w)) \psi(w)[1-\psi(w)]
$$

for $w \in \mathscr{O}_{d}$. Then $\tilde{f} \in B\left(\mathscr{Q}_{d}\right)$ provided

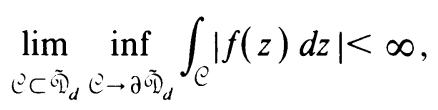

where $\mathcal{C}$ is some curve; we obtain (2.12) from (2.2) and (2.3) by applying the change of variables $z=\psi(w)$ to the integrals in (2.2) and (2.3).

Suppose, for example, $f$ has singularities at $z=0$ and at $z=1$, but $f$ is analytic in $\tilde{\mathscr{D}}_{d}$, and $f$ is continuous on $\partial \tilde{\mathscr{Q}}_{d} \backslash\{0,1\}$. Then, by examining the integrals over portions of the curves $\bigodot$ near $z=0$ and $z=1$ and the integrals over portions of $巳$ away from $z=0$ and $z=1$ separately, one can show (2.12) holds if and only if

$$
\int_{\partial \tilde{\mathscr{D}}_{d}}|f(z) d z|<\infty
$$

Suppose further that $f$ is such that we may take $d=\pi / 2$, so $\partial \tilde{\mathscr{D}}_{d}=\left\{z:\left|z-\frac{1}{2}\right|=\frac{1}{2}\right\}$. Then $\psi$ maps the line $\{z: \operatorname{Im}(z)=\pi / 2\}$ to the semicircle $\left\{\left|z-\frac{1}{2}\right|=\frac{1}{2}, \operatorname{Im}(z)>0\right\}$ and the line $\{z: \operatorname{Im}(z)=-\pi / 2\}$ to the semicircle $\left\{\left|z-\frac{1}{2}\right|=\frac{1}{2}, \operatorname{Im}(z)<0\right\}$. Condition (2.12) then becomes

$$
\begin{aligned}
& \int_{\mathcal{C}}|f(z) d z|=\frac{1}{2} \int_{0}^{2 \pi}\left|f\left(\frac{1}{2}+\frac{1}{2} e^{i t}\right)\right| d t \\
& \quad=\int_{\pi / 4}^{3 \pi / 4}\left|f\left(e^{i u} \cos (u)\right)\right| d u+\int_{-\pi / 4}^{\pi / 4}\left|f\left(1-e^{i v} \cos (v)\right)\right| d v<\infty .
\end{aligned}
$$

Condition (2.12) then holds for those $f$ which satisfy

(2.13) $f(z)=O\left(|z|^{\alpha_{0}}\right)$ as $z \rightarrow 0$, and $f(z)=O\left(|1-z|^{\alpha_{1}}\right)$ as $z \rightarrow 1$,

where $\alpha_{0}>-1$ and $\alpha_{1}>-1$, and which are continuous elsewhere on the closure of $\tilde{\mathscr{D}}_{d}$ and which are analytic in $\tilde{\mathscr{D}}_{d}$.

Corresponding to Theorem 2.1, we have

THEOREM 2.3. Suppose $f$ is analytic in some $\tilde{D}_{d}$ as in (2.9), suppose $f$ satisfies (2.12) ( or $\left.(2.12)^{\prime}\right)$, suppose

$$
|f(x)| \leqslant C x^{\beta}(1-x)^{\beta}, \quad 0<x<1,
$$

for some $\beta>-1$ and some $C$ dependent on $f$ and set $h=[\pi d / \alpha N]^{1 / 2}$, where $\alpha=\beta+1$. Then

$$
\begin{gathered}
\left\|f(x)-\sum_{k=-N}^{N} f(\psi(k h)) \psi(k h)[1-\psi(k h)] S(k, h)(\varphi(x))\right\|_{\infty} \\
\leqslant C^{\prime} N^{1 / 2} e^{-(\pi d \alpha N)^{1 / 2}}
\end{gathered}
$$

for some $C^{\prime}$ dependent on $f$ but not on $N$. 
Similarly, we have

THEOREM 2.4. Suppose $f$ is analytic in $\tilde{\mathrm{T}}_{d}$ for some $d$, suppose $f$ satisfies (2.12) (or $\left.(2.12)^{\prime}\right)$, suppose $f$ satisfies $(2.5)^{\prime}$ for some $\beta>-1$ and some $C$ dependent on $f$, and set $h^{\prime}=[2 \pi d / \alpha N]^{1 / 2}$. Then

$$
\left|\int_{0}^{1} f(t) d t-h^{\prime} \sum_{k=-N}^{N} f\left(\psi\left(k h^{\prime}\right)\right) \psi\left(k h^{\prime}\right)\left[1-\psi\left(k h^{\prime}\right)\right]\right| \leqslant C_{I} e^{-(2 \pi d \alpha N)^{1 / 2}}
$$

for some $C_{I}$ dependent on $f$ but not on $N$.

3. The Indefinite Integral Approximation Formulas. Analogously to Section 2, we will first obtain an approximation procedure for integrals of the form

$$
F(w)=\int_{-\infty}^{w} f(t) d t,
$$

where $f \in B\left(\mathscr{D}_{d}\right)$ (cf. Definition 2.1). We will then use the conformal mapping procedure to obtain approximation rules for integrals of the form

$$
F(x)=\int_{\psi(-x)}^{\psi(x)} f(\varphi(t)) d t,
$$

where $\varphi$ and $\psi$ can be as in formulas (2.8) and (2.10), respectively. We begin with the following lemma, which is a direct consequence of Theorem 2.1.

Lemma 3.1. Define $F(w)$ as in (3.1), suppose $\left.F \in B_{p}\left({ }^{(1)}\right)_{d}\right)$, for some $p \in[1, \infty)$, suppose $|F(w)| \leqslant C e^{-\alpha|w|}$ for some $C$ and $\alpha>0$ and all $w \in \mathbf{R}$, and set $h=$ $[\pi d /(\alpha N)]^{1 / 2}$. Then

$$
\left|\int_{-\infty}^{n} f(t) d t-\left\{\sum_{k=-N}^{N}\left[\int_{-\infty}^{k h} f(t) d t\right] S(k, h)(w)\right\}\right| \leqslant C_{I}^{\prime} N^{1 / 2} e^{(\pi d \alpha N)^{1 / 2}}
$$

for all $w \in \mathbf{R}$ and for some indefinite integral approximation constant $C_{I}^{\prime}$ which does not depend on $N$.

Lemma 3.1 does not apply to general integrals $F$ as in (3.1), since in general $\int_{-\infty}^{\infty} f(t) d t \neq 0$, and formula (2.5) is not valid. For such $F$, we consider the related function

$$
\begin{aligned}
G(w) & =\int_{-\infty}^{u} f(t) d t-\frac{e^{\alpha w / 2}}{e^{-\alpha w^{\prime} / 2}+e^{\alpha w / 2}} \int_{-\infty}^{\infty} f(t) d t \\
& =\int_{-\infty}^{u^{u}}\left[f(t)-\frac{\alpha e^{\alpha t}}{\left(e^{\alpha t}+1\right)^{2}} I_{\infty}(f)\right] d t=\int_{-\infty}^{u^{\prime}} g(t) d t,
\end{aligned}
$$

where

$$
g(t)=f(t)-\frac{\alpha e^{\alpha t}}{\left(e^{\alpha t}+1\right)^{2}} I_{\infty}(f), \quad I_{\infty}(f)=\int_{-\infty}^{\infty} f(t) d t .
$$

We then have

Lemma 3.2. Suppose $|f(w)| \leqslant C e^{-\alpha|w|}$ for some $C$ and $\alpha>0$ and all $w \in \mathbf{R}$, and suppose $G$ is as in (3.3). Then there is a $C_{G}$ such that $|G(w)| \leqslant C_{G} e^{-\alpha|w|}$ for all $w \in \mathbf{R}$. 
Proof. We have

$$
\begin{aligned}
|G(w)| \leqslant & \left|\frac{e^{\alpha w / 2}}{e^{\alpha w / 2}+e^{-\alpha w / 2}}\left[\int_{-\infty}^{w} f(t) d t-I_{\infty}(f)\right]\right| \\
& +\left|\frac{e^{-\alpha w / 2}}{e^{\alpha w / 2}+e^{-\alpha w / 2}} \int_{-\infty}^{w} f(t) d t\right|
\end{aligned}
$$

We will consider each factor in the two terms on the right as $w \rightarrow-\infty$ and as $w \rightarrow+\infty$.

Case $1, w \rightarrow-\infty$. The second factor in the first term is bounded and approaches $-I_{\infty}(f)$, while the first factor in the first term is bounded by $e^{-\alpha|w|}$. The first factor in the second term is bounded by 1 , while the second factor in the second term is bounded by $C e^{-\alpha|w|} / \alpha$.

Case 2, $w \rightarrow \infty$. The first factor in the first term is bounded by 1 , while the second factor in the first term is equal to $-\int_{w}^{\infty} f(t) d t$ and hence is bounded by $C e^{-\alpha|w|} / \alpha$. The first factor in the second term is bounded by $e^{-\alpha|w|}$, while the second factor in the second term is bounded and approaches $I_{\infty}(f)$.

Combining the above, we may take

$$
\begin{aligned}
C_{G} & =C / \alpha+\max \left\{\sup _{w \leqslant 0}\left|\int_{-\infty}^{w} f(t) d t-I_{\infty}(f)\right|, \sup _{w \geqslant 0}\left|\int_{-\infty}^{w} f(t) d t\right|\right\} \\
& \sim C / \alpha+\left|I_{\infty}(f)\right| .
\end{aligned}
$$

Combining Lemma 3.1 and Lemma 3.2 immediately gives

Theorem 3.1. Suppose $f \in B\left(\mathscr{D}_{d}\right)$, suppose $|f(w)| \leqslant C e^{-\alpha|w|}$ for some $C$ and all $w \in \mathbf{R}$, and suppose $G \in B_{p}\left(\mathscr{Q}_{d}\right)$ for some $p$, where $G$ is as in (3.3). Define

$$
\begin{aligned}
F_{N}(w)= & \sum_{k=-N}^{N}\left\{\int_{-\infty}^{k h}\left[f(t)-\frac{\alpha e^{\alpha t}}{\left(e^{\alpha t}+1\right)^{2}} \int_{-\infty}^{\infty} f(u) d u\right] d t\right\} S(k, h)(w) \\
& +\frac{e^{\alpha w / 2}}{e^{-\alpha w / 2}+e^{\alpha w / 2}} \int_{-\infty}^{\infty} f(t) d t
\end{aligned}
$$

where $h=[\pi d /(\alpha N)]^{1 / 2}$. Then there is a $C_{I}^{\prime}$, independent of $N$ such that

$$
\left|\int_{-\infty}^{w} f(t) d t-F_{N}(w)\right| \leqslant C_{I}^{\prime} N^{1 / 2} e^{-(\pi d \alpha N)^{1 / 2}}
$$

for all $w \in \mathbf{R}$.

In practice the quantities

$$
\int_{-\infty}^{k h}\left[f(t)-\frac{\alpha e^{\alpha t}}{\left(e^{\alpha t}+1\right)^{2}} \int_{-\infty}^{\infty} f(u) d u\right] d t=\int_{-\infty}^{k h} g(t) d t=G(k h),
$$

and the quantity $\int_{-\infty}^{\infty} f(u) d u=I_{\infty}(f)$ must be approximated. Below, we show how to do this so that the order of approximation in (3.5) remains $O\left(N^{2} e^{-(\pi d \alpha N)^{1 / 2}}\right)$.

First observe that linear combinations of functions in $B\left(\mathscr{D}_{d}\right)$ are also in $B\left(\mathscr{D}_{d}\right)$; also linear combinations of functions satisfying (2.5) satisfy (2.5) (with a different $C)$. Thus, we deduce 
LEMMA 3.3. Suppose $f \in B\left(\mathscr{O}_{d}\right)$, suppose $|f(w)| \leqslant C e^{-\alpha|w|}$ for some $C$ and $\alpha>0$ and all $w \in \mathbf{R}$, and suppose $g$ is as in (3.4). Then $g \in B\left(\mathscr{Q}_{d}\right)$, and there is a $C_{g}$ such that $|g(w)| \leqslant C_{g} e^{-\alpha|w|}$ for all $w \in \mathbf{R}$. Thus, $g$ and $\int_{-\infty}^{\infty} g(t) d t$ may be approximated as in (2.6) and (2.7), respectively.

Let $C_{N}(g, h)(t)=\sum_{j=-N}^{N} g(j h) S(j, h)(t)$ be the approximation to $g$. We use $C_{N}(g, h)(t)$ to approximate the quantities $G(k h)$ in (3.5).

Lemma 3.4. Let $f \in B\left(\mathcal{Q}_{d}\right)$, suppose $|f(t)| \leqslant C e^{-\alpha|t|}, t \in \mathbf{R}$, and set $h=$ $[\pi d /(\alpha N)]^{1 / 2}$. Suppose $g$ and $G$ are as in (3.3) and (3.4), and define

$$
\hat{G}_{N}(w)=\int_{-N h}^{n} C_{N}(g, h)(t) d t=\sum_{j=-N}^{N} g(j h) \int_{-N h}^{n} S(j, h)(t) d t,
$$

where $-N h \leqslant w \leqslant N h$. Then there is a constant $\hat{C}$ dependent on $f$ such that $\mid \hat{G}_{N}(w)-$ $G(w) \mid \leqslant \hat{C} N e^{-(\pi d \alpha N)^{1 / 2}}$ for all $w \in \mathbf{R}$.

Proof.

$$
\left|\hat{G}_{N}(w)-G(w)\right| \leqslant|G(-N h)|+\int_{-N h}^{w}\left|g(t)-C_{N}(g, h)(t)\right| d t .
$$

But $|G(-N h)| \leqslant C_{G} e^{-\alpha N h}=C_{G} e^{-(\pi d \alpha N)^{1 / 2}}$, from Lemma 3.2. Also

$$
\begin{aligned}
\int_{-N h}^{w^{*}}\left|g(t)-C_{N}(g, h)(t)\right| d t & \leqslant(N H+w) C_{g}^{\prime} N^{1 / 2} e^{-(\pi d \alpha N)^{1 / 2}} \leqslant 2 N^{3 / 2} h C_{g}^{\prime} e^{-(\pi d \alpha N)^{1 / 2}} \\
& =\tilde{C} N e^{-(\pi d \alpha N)^{1 / 2}},
\end{aligned}
$$

from Lemma 3.3. Thus

$$
\left|\hat{G}_{N}(w)-G(w)\right| \leqslant C_{G} e^{-(\pi d \alpha N)^{1 / 2}}+\tilde{C} N e^{-(\pi d \alpha N)^{1 / 2}} \leqslant \hat{C} N e^{-(\pi d \alpha N)^{1 / 2}}
$$

for some appropriate $\hat{C}$.

We now replace $G(k h)$ by $\hat{G}(k h)$ in Theorem 3.1 .

LEMMA 3.5. Suppose the assumptions of Theorem 3.1 hold, but

$$
G(k h)=\int_{-\infty}^{k h}\left[f(t)-\frac{\alpha e^{\alpha t}}{\left(e^{\alpha t}+1\right)^{2}} \int_{-\infty}^{\infty} f(u) d u\right] d t
$$

in (3.5) is replaced by $\hat{G}_{N}(k h)$ as in (3.6), to obtain

(3.5)' $\quad \hat{F}_{N}(w)=\sum_{k=-N}^{N} \hat{G}_{N}(k h) S(k, h)(w)+\frac{e^{\alpha w / 2}}{e^{-\alpha w / 2}+e^{\alpha w / 2}} \int_{-\infty}^{\infty} f(t) d t$.

Then there is a $\hat{C}_{F}$ independent of $N$ such that

$$
\left|\int_{-\infty}^{w} f(t) d t-\hat{F}_{N}(w)\right| \leqslant \hat{C}_{F} N^{2} e^{-(\pi d \alpha N)^{1 / 2}}
$$

Proof.

$$
\left|\int_{-\infty}^{w} f(t) d t-\hat{F}_{N}(w)\right| \leqslant\left|\int_{-\infty}^{w} f(t) d t-F_{N}(w)\right|+\left|F_{N}(w)-\hat{F}_{N}(w)\right|,
$$


where the first term on the right is bounded by $C_{I}^{\prime} N^{1 / 2} e^{-(\pi d \alpha N)^{1 / 2}}$, by Theorem 3.1. On the other hand,

$$
\begin{aligned}
\left|F_{N}(w)-\hat{F}_{N}(w)\right| & =\left|\sum_{k=-N}^{N}\left[G(k h)-\hat{G}_{N}(k h)\right] S(k, h)(w)\right| \\
& \leqslant \sum_{k=-N}^{N}\left|G(k h)-\hat{G}_{N}(k h)\right| \leqslant 2 N\left(\hat{C} N e^{-(\pi d \alpha N)^{1 / 2}}\right) \\
& \leqslant \hat{C}_{F} N^{2} e^{-(\pi d \alpha N)^{1 / 2}}
\end{aligned}
$$

for some constant $\hat{C}_{F}$ dependent on $f$ but not on $N$.

For the main result, we must replace $I_{x}(f)=\int_{-\infty}^{\infty} f(u) d u$ in (3.5)' without changing the order of approximation.

Lemma 3.6. Suppose the assumptions of Theorem 3.1 hold, but in (3.5)' we replace $I_{\infty}(f)=\int_{-\infty}^{\infty} f(t) d t$ by $I_{N}(f)=h \sum_{k=-N}^{N} f(k h)$, where $h=[\pi d /(\alpha N)]^{1 / 2}$. Then the conclusion of Lemma 3.5 is still true.

Proof. If there are a $C$ and an $\alpha>0$ such that $|f(t)| \leqslant C e^{-\alpha|t|}, t \in \mathbf{R}$, then, if $\alpha^{\prime}=\alpha / 2,|f(t)| \leqslant C e^{-\alpha^{\prime}|t|}, t \in \mathbf{R}$. Thus from Theorem 2.2.

$$
\left|I_{\infty}(f)-I_{N}(f)\right| \leqslant C_{I} e^{-(\pi d \alpha N)^{1 / 2}} .
$$

Define

$$
\tilde{g}_{N}(t)=\sum_{j=-N}^{N}\left[f(j h)-\frac{\alpha e^{\alpha j h}}{\left(e^{\alpha j h}+1\right)^{2}} I_{N}(f)\right] S(j, h)(t),
$$

and define

$$
\tilde{G}_{N}(w)=\int_{-N h}^{n} C_{N}\left(\tilde{g}_{N}, h\right)(t) d t
$$

and

$$
\tilde{F}_{N}(w)=\sum_{k=-N}^{N} \tilde{G}_{N}(k h) S(k, h)(w)+\frac{e^{\alpha w / 2}}{e^{-\alpha w / 2}+e^{\alpha w / 2}} I_{N}(f)
$$

Then

$$
\left|\int_{-\infty}^{u} f(t) d t-\tilde{F}_{N}(w)\right| \leqslant\left|\int_{-\infty}^{n} f(t)-\hat{F}_{N}(w)\right|+\left|\hat{F}_{N}(w)-\tilde{F}_{N}(w)\right|
$$

where $\hat{F}_{N}$ is as in $(3.5)^{\prime}$. By Lemma 3.5, the first term on the right of (3.10) is bounded by $\hat{C}_{F} N^{2} e^{-(\pi d \alpha N)^{1 / 2}}$, while

$$
\begin{aligned}
\left|\hat{F}_{N}(w)-\tilde{F}_{N}(w)\right| \leqslant\left|\sum_{k=-N}^{N}\left[\hat{G}_{N}(k h)-\tilde{G}_{N}(k h)\right] S(k, h)(w)\right| \\
+\frac{e^{\alpha w / 2}}{e^{-\alpha w / 2}+e^{\alpha w / 2}}\left|I_{\infty}(f)-I_{N}(f)\right|,
\end{aligned}
$$


where $\hat{G}_{N}$ is as in (3.6). The first term on the right of (3.11) equals

$$
\left|\sum_{k=-N}^{N}\left\{\sum_{j=-N}^{N} \frac{\alpha e^{\alpha j h}}{\left(e^{\alpha j h}+1\right)^{2}}\left[I_{N}(f)-I_{x}(f)\right] \int_{-N h}^{k h} S(j, h)(t) d t\right\} S(k, h)(w)\right|,
$$

while the inner sum in (3.12) equals

$$
\Lambda_{k, N}=h \sum_{j=-N}^{N} \frac{\alpha e^{\alpha j h}}{\left(e^{\alpha j h}+1\right)^{2}}\left[I_{N}(f)-I_{\infty}(f)\right] \int_{-N-j}^{k-j} \frac{\sin (\pi t)}{\pi t} d t .
$$

But $\Lambda_{k, N}$ is a trapezoidal rule approximation, as in Theorem 2.2, to $\int_{-\infty}^{\infty} \lambda_{k, N}(t) d t$, where

$$
\begin{aligned}
\lambda_{k, N}(w) & =\left[I_{N}(f)-I_{x}(f)\right]\left[\frac{\alpha e^{\alpha w}}{\left(e^{\alpha w}+1\right)^{2}}\right] \int_{-N-w / h}^{k-w / h} \frac{\sin (\pi t)}{\pi t} d t \\
& =\frac{\alpha\left[I_{N}(f)-I(f)\right]}{4}\left[\int_{-N-w / h}^{k-w / h} \frac{\sin (\pi t)}{\pi t} d t\right] \operatorname{sech}^{2}\left[\left(\frac{\alpha}{2}\right) w\right],
\end{aligned}
$$

where $h^{\prime}=(\pi d / \alpha N)^{1 / 2}=\left(2 \pi d / \alpha^{\prime} N\right)^{1 / 2}$ for $\alpha^{\prime}=\alpha / 2$. But the magnitude of the integral in (3.13) is bounded by

$$
\frac{2}{\pi} \sup _{a \geqslant 0} \int_{0}^{a} \frac{\sin (t)}{t} d t<2
$$

thus

$$
\left|\lambda_{k, N}(w)\right| \leqslant \frac{2 \alpha\left|I_{N}(f)-I_{\infty}(f)\right|}{\left(e^{\alpha w / 2}+e^{-\alpha w / 2}\right)^{2}} \leqslant 2 \alpha\left|I_{N}(f)-I_{\infty}(f)\right| e^{-\alpha|w|} .
$$

It is also possible to verify that $\lambda_{k, N}(w) \in B\left(\mathscr{D}_{d}\right)$. Thus, as in Theorem 2.2,

$$
\left|\int_{-\infty}^{\infty} \lambda_{k, N}(t) d t-\Lambda_{k, N}\right| \leqslant C_{I}^{\Lambda} e^{-(\pi d \alpha N)^{1 / 2}}
$$

for some constant $C_{I}^{\Lambda}$ dependent on $f$ but not on $N$. But, by (3.7)

$$
\begin{aligned}
\left|\int_{-\infty}^{\infty} \lambda_{k, N}(t) d t\right| & \leqslant \frac{\alpha}{4}\left|I_{N}(f)-I_{\infty}(f)\right|\left\{\int_{-\infty}^{\infty} \operatorname{sech}^{2}\left[\left(\frac{\alpha}{2}\right) t\right] d t\right\} \\
& =\left|I_{N}(f)-I_{\infty}(f)\right| \leqslant C_{I} e^{-(\pi d \alpha N)^{1 / 2}} .
\end{aligned}
$$

Combining (3.17) and (3.16), we see that the quantity in (3.12) is bounded by

$$
\sum_{k=-N}^{N}\left(C_{I}+C_{I}^{\Lambda}\right) e^{-(\pi d \alpha N)^{1 / 2}}=2\left(C_{I}+C_{I}^{\Lambda}\right) N e^{-(\pi d \alpha N)^{1 / 2}}
$$

Combining the bounds (3.18), (3.7), and (3.11) now gives

$$
\left|\hat{F}_{N}(w)-\tilde{F}_{N}(w)\right| \leqslant 2\left(C_{I}+C_{I}^{\Lambda}\right) N e^{-(\pi d \alpha N)^{1 / 2}}+C_{I} e^{-(\pi d \alpha N)^{1 / 2}} .
$$

We obtain the conclusion in Lemma 3.6 from Lemma 3.5, 3.10, and 3.19. 
We summarize the above in the following

Theorem 3.2 (MAIN Result). Suppose $f \in B\left(\mathscr{D}_{d}\right)$, and $|f(w)| \leqslant C e^{-\alpha|w|}$ for some $\alpha>0$ and $C$, and all $w \in \mathbf{R}$. Suppose further $G \in B\left(\mathscr{D}_{d}\right)$, where $G$ is defined in terms of $f$ by (3.3). Define $\sigma_{q}, q$ an integer, by

$$
\sigma_{q}=\int_{0}^{q} \frac{\sin (\pi t)}{\pi t} d t .
$$

Set $h=[\pi d /(\alpha N)]^{1 / 2}$, and approximate $\int_{-\infty}^{w} f(t) d t$ by

$$
\begin{aligned}
\tilde{F}_{N}(w)= & h\left[\sum_{k=-N}^{N}\left\{\sum_{j=-N}^{N}\left[f(j h)-\frac{\alpha e^{\alpha j h}}{\left(e^{\alpha j h}+1\right)^{2}} I_{N}(f)\right]\left(\sigma_{N+j}+\sigma_{k-j}\right)\right\} S(k, h)(w)\right] \\
& +\frac{e^{\alpha w / 2}}{e^{-\alpha w / 2}+e^{\alpha w / 2}} I_{N}(f),
\end{aligned}
$$

where $I_{N}(f)=h \sum_{k=-N}^{N} f(k h)$. Then there is a constant $C_{F}$ independent of $N$ such that

$$
\left|\int_{-\infty}^{u} f(t) d t-\tilde{F}_{N}(w)\right| \leqslant C_{F} N^{2} e^{-(\pi d \alpha N)^{1 / 2}}
$$

for all $w \in \mathbf{R}$.

Proof. Theorem 3.2 is a restatement of Lemma 3.6, once one observes

$$
h\left(\sigma_{N+j}+\sigma_{k-j}\right)=\int_{-N h}^{k h} S(j, h)(t) d t .
$$

A table of $\sigma_{q}, q=1, \ldots, 100$, is found on p. 175 of [4]. Also, $\sigma_{q}=\operatorname{Si}(q \pi) / \pi$, an asymptotic expansion for which is given on p. 244 of [1]; we are told this expansion gives 20 significant figures for $q \geqslant 20$.

Theorem 3.2 is used in conjunction with conformal mapping techniques (explained in [4, Section 4], and the references therein) to evaluate singular integrals of the form $\int_{a}^{b} f(t) d t$, where $a$ or $b$ or both $a$ and $b$ are finite, and $f$ possibly has singularities at $a$ or $b$. We consider here $(a, b)=(0,1)$, and the conformal maps $\varphi$ and $\psi$ defined in (2.8) and (2.10). From Theorem 3.2 and considerations in Section 2 of this paper, we obtain

ThEOREM 3.3. Suppose (as in (2.8)-(2.13)) that $f \in B\left(\tilde{\mathscr{D}}_{d}\right)$, and suppose

$$
|f(x)| \leqslant C|x|^{\beta}(1-x)^{\beta} \text { all } x \in(0,1),
$$

for some' (' and $\beta>-1$. Set $\alpha=\beta+1$, and set $h=(\pi d / \alpha N)^{1 / 2}$. Define

$$
\begin{array}{r}
\tilde{F}_{N}(x)=h\left[\sum_{k=-N}^{N}\left\{\sum_{j=-N}^{N}\left[f\left(x_{j}\right)\left(x_{j}\right)\left(1-x_{j}\right)-\frac{\alpha e^{\alpha j h}}{\left(e^{\alpha j h}+1\right)^{2}} I_{N}(f)\right]\left(\sigma_{N+j}+\sigma_{k-j}\right)\right\}\right. \\
\left.\cdot S(k, h)(\varphi(x))+\frac{x^{\alpha}}{x^{\alpha}+(1-x)^{\alpha}} I_{N}(f)\right],
\end{array}
$$


where $I_{N}(f)=h \sum_{k=-N}^{N} f\left(x_{k}\right)\left(x_{k}\right)\left(1-x_{k}\right)$ and where $x_{j}=\psi(j h)=e^{j h} /\left(1+e^{j h}\right)$. Then, for $x \in(0,1)$,

$$
\left|\int_{0}^{x} f(t) d t-\tilde{F}_{N}(x)\right| \leqslant C_{F} N^{2} e^{-(\pi d \alpha N)^{1 / 2}}
$$

for some $C$ dependent on $f$ but independent of $N$. If $f(z)=O\left(|z|^{\beta}\right)$ as $z \rightarrow 0, z \in \mathbf{C}$, then $f \in B\left(\tilde{\mathscr{Q}}_{d}\right)$, with $d=\pi / 2$.

Note that (3.22) is equivalent to

$$
|f(\psi(w)) \psi(w)[1-\psi(w)]|=|f(x) x(1+x)| \leqslant c e^{-\alpha|w|} \text { for } w \in \mathbf{R} .
$$

Theorem 3.3. is otherwise obtained from Theorem 3.2 by making the transformation

$$
\int_{0}^{x} f(t) d t=\int_{-\infty}^{\varphi(x)} f(\psi(u)) \psi(u)[1-\psi(u)] d u,
$$

then applying Theorem 3.2 to the right member of (3.24).

To comment on the power of the approximations in Theorem 3.2 and Theorem 3.3, we note that it is unnecessary to know precisely what the order $\alpha$ is; in particular, if $|f(t)| \leqslant c e^{-\alpha t}$ and $0<\alpha^{\prime}<\alpha$, then we may replace $\alpha$ by $\alpha^{\prime}$ in any of the formulas. Note that the convergence order is not preserved in this way when we use, say, Newton-Cotes or Gauss type formulas derived with the method of moments for weighted integrands of the form $x^{\alpha} p(x), p$ a polynomial. Also, sinc function approximations are optimal, as explained in [3].

4. Experimental Results. To empirically test formula (3.21)', we tried it for various $N$ on the following functions:

(i) $f_{1}(x)=\frac{1}{3} x^{-2 / 3}$;

(ii) $f_{2}(x)=\frac{4}{3} x^{1 / 3}$;

(iii) $f_{3}(x)=\frac{1}{6}\left[x^{-2 / 3}+(1-x)^{-2 / 3}\right]$; and

(iv) $f_{4}(x)=\frac{3}{40}\left[x^{-.9}+(1-x)^{-.7}\right]$.

In each case, the difference $\tilde{F}_{N}(x)-\int_{0}^{x} f(t) d t$ was computed at $x=.1 i, i=1, \ldots, 10$, for $N=4,8,16$, and 32, corresponding to 9,17,33, and 65 evaluations of $f$, respectively. These results were compared to the errors $F_{N}^{G}(x)-\int_{0}^{x} f(t) d t$, where $F_{N}^{G}(x)$ is the result of applying a $2 N$-point Gauss-Legendre formula to compute $\int_{0}^{x} f(t) d t$, for $2 N=8,16$, and $32 ; F_{64}^{G}(x)$ was defined to be the result of applying the composite 32-point Gauss-Legendre formula with 2 subdivisions. These particular formulas were chosen for comparison since the Gauss formulas are commonly thought to be generally accurate, and since the 8-, 16-, and 32-point formulas were closest in number of points to 9,17 , and 33 of those formulas already programmed on our system.

The functions $f_{i}, i=1, \ldots, 4$, are normalized so that $\int_{0}^{1} f_{i}(t) d t=1$. The functions have singularities at $x=0$ and $x=1$ of varying orders; the singularities of $f_{3}$ are the same order at $x=0$ and $x=1$, while the singularities of $f_{i}, i \neq 3$, differ in order at $x=0$ and $x=1$.

Four tables appear, corresponding to each of the four functions. Table 3 has three parts, corresponding to various estimated orders $\beta$ (cf. (3.22)).

The sinc function approximations compare favorably to the corresponding Gauss formulas, except for $f_{2}(x)$. We postulate that the worse the singularity, the better the merit of the sinc function approximation. 
mmmmmmmmm

1000000000

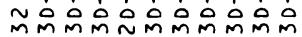
" 0000000000 ○ं

с

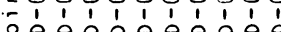

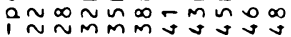

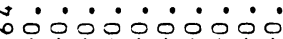

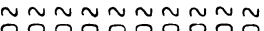
$\begin{array}{llll}1 & 1 & 1 & 1 \\ 0 & 1 & 1 & 1\end{array}$

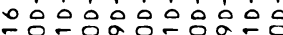
" ipipiịiii

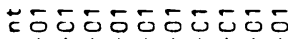

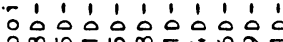
in $\sim$ m mpipipipoji

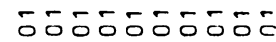
11111111111111 " $\sim \bar{\sim} \sim \bar{\sim} \sim \sim \bar{\sim} \sim \bar{\sim}$

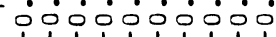

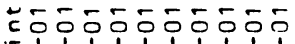

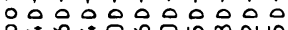
1 v n -

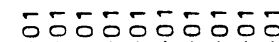
- 10.11190 .11 " nnnun nununu ipisipipi

-

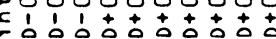

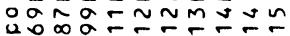

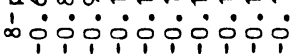

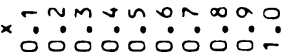

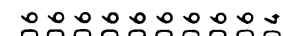

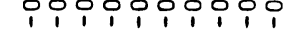
$\sim 0 \infty 000000000$

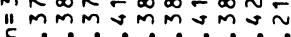
iopipioji

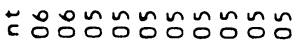
$=11101011$ o o o o o o o o o o iñoㄷำ ○

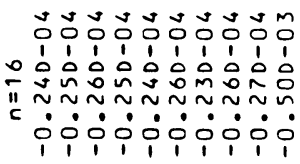

ㅇํำกำกำกับง 1111

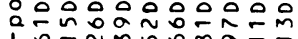

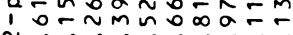
mं00ல0ல0ல0ல

vim

11

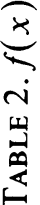

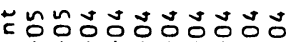

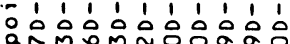

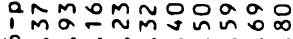
- 000000000

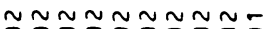

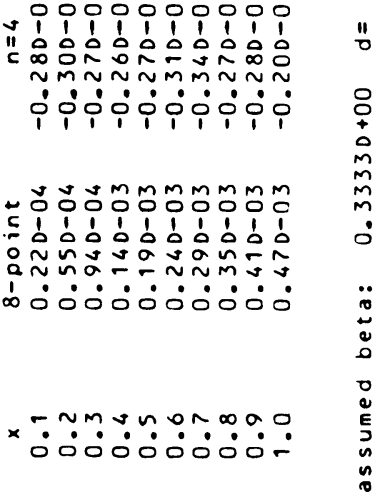




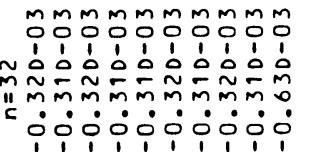

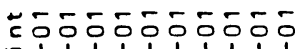

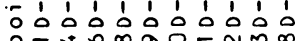
II $\div \infty a 0 \approx \approx m \infty$ -

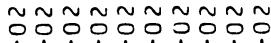
O - $\begin{aligned} & 0 \\ & 0\end{aligned}$

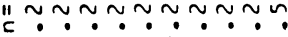
1 1 1 1000000

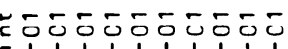
영ㅇㅇㅇㅇㅇㅇㅇㅇㅇ응 $1 \div \infty \sim \approx \sim \sim \sim \sim \sim ⿻ 上$ N

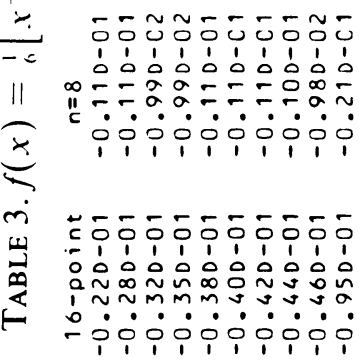

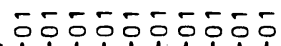

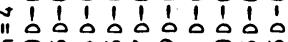

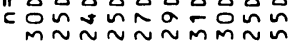

icipisioisio

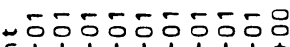

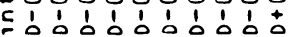

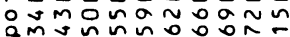
占

×

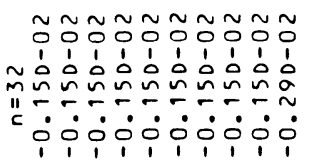

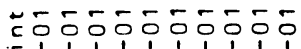

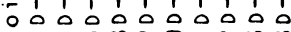
a- 0 -

ก $\sim \sim \sim \sim \sim \sim \sim \sim-$ OPOPOOPOOPOO

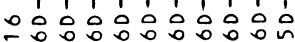

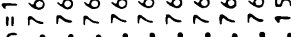

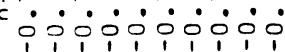

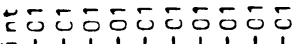
-

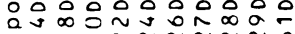
1-r n N N N N 0

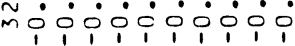

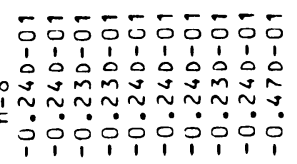

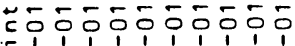

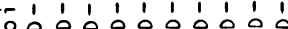

$i \approx \sim \infty \sim ⿻ 上 丨$

-

둥ㅎㅇㅎㄷㅇ응

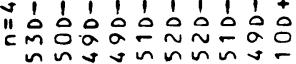
نं

-

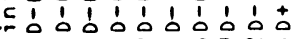

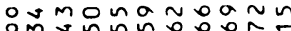

官

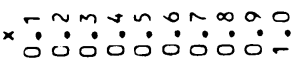

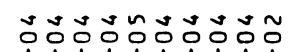

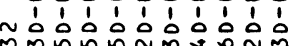
mmñ -

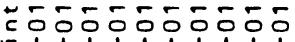

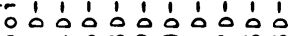

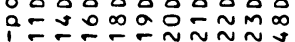

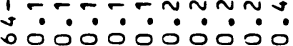
YiPOPOPOPO

mómmmmmm opopoopooo o 0 d d $\begin{aligned} & 1 \\ & 0\end{aligned}$

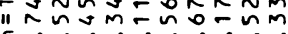
-

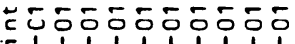

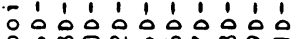

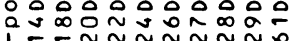
míi

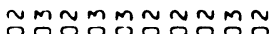

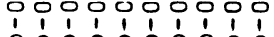

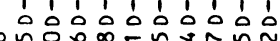
" $\approx a-\infty \sigma \tilde{m} \sim \tilde{N}$ -

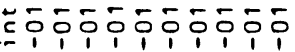

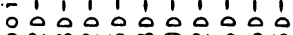
우요 -

5
+
$\vdots$
$\tilde{n}$
$\vdots$
0

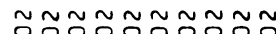

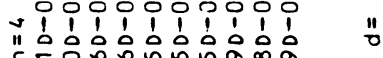

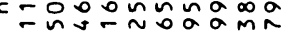

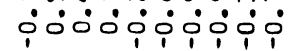

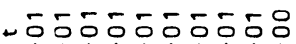

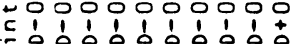
ommuññ

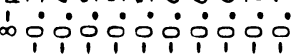

0
0
+
0
0
0
0
0
1
0
0
0
0

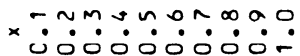



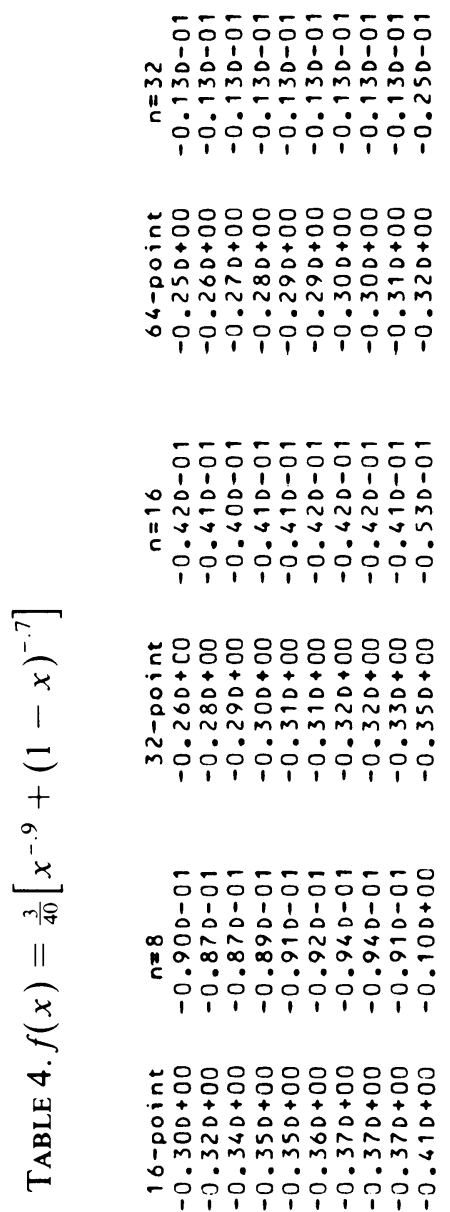

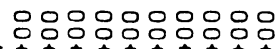

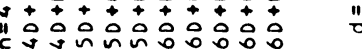

ióicióió

- 880880888880

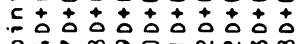

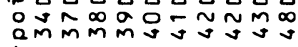
sipipipiói

5
+
0
0
0
0
0
0
0
0
0
$\vdots$
0
0
0
0
1
$\ddot{0}$
0
0
0

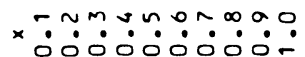


For Table $1, \beta=-2 / 3(\alpha=1 / 3)$ was used, while $\beta=1 / 3$ in Table 2 . The values $\beta=-2 / 3, \beta=-.467$, and $\beta=-.867$ were used in Tables $3 \mathrm{a}, 3 \mathrm{~b}$, and $3 \mathrm{c}$, respectively, while the value $\beta=-.9$ was used in Table 4 . From these and other experiments, it appears it is better to underestimate $\beta$ than to overestimate it.

In all cases, $d$ was taken to be $\pi / 2$.

Stenger has proven a formula analogous to $(3.5)^{\prime}$, namely

$$
\int_{-\infty}^{x} g(t) d t=h \sum_{k=-N}^{N} \sum_{l=-N}^{N} \sigma_{k-l} g(l h) S(k, h)(x)+O\left(N e^{-(\pi d \alpha N)^{1 / 2}}\right),
$$

using formulas (2.17), (3.7), and (3.11) in [4] and the uniqueness of the coefficients in the sinc function expansion [5]. (This formula appears without proof as formula (3.36) in [4]*.) It is then possible, using the same argument as in the proof of Lemma 3.6, to obtain an analogue to Lemma 3.6 from (4.1). This analogue was also tried in the same cases as above; in all cases, the tables of errors were the same to at least two significant digits. Since (4.1) is more elegant and requires less additions, it is perhaps to be preferred.

The formula resulting from (3.21) when $I_{N}$ is replaced by $I_{\infty}$ was also tried. From the resulting error tables, it appeared that the approximation for $I_{x}$ accounted for approximately half of the total error. Thus, it seems unnecessary to use a separate stepsize $h$ in computing $I_{N}$, as suggested by (2.7). Also, taking $d$ other than $\pi / 2$ in no case improved the performance.

The computations were done on a Honeywell 6880 (Multics system) in double precision (63-bit mantissas). To get values at $x=1, \tilde{x}=1-2^{-62}$ was used.

Acknowledgements. I wish to thank Professor Frank Stenger for introducing me to these approximation techniques. I also wish to thank the referee for his careful reading and for his suggestions in making the paper more comprehensible and useful.

Department of Mathematics and Statistics

University of Southwestern Louisiana

Lafayette, Louisiana 70504

1. M. Abramowitz \& I. A. Stegun, Editors, Handbook of Mathematical Functions, Dover, New York, 1970.

2. F. Stenger, “Approximations via Whittaker's cardinal function,” J. Approx. Theory, v. 17, 1976, pp. $222-240$.

3. F. STENGER, “Optimal convergence of minimum norm approximations in $H^{p}$ ", Numer. Math., v. 29. 1978, pp. 345-362.

4. F. STENGER, "Numerical methods hased on Whittaker cardinal, or sinc functions," SIAM Rev., v. 23, 1981, pp. 165-224.

5. F. STENGER, private communication.

${ }^{*}$ Formula (3.36) in [4] was attributed to Kearfott, Sikorski, and Stenger; that paper never existed. Also, the derived formula (4.58) in [4] was mistakenly attributed to John Lund; that formula corresponds to (3.23) of this paper. 